\title{
Fouling and cleaning of seawater reverse osmosis membranes in Kalpakkam nuclear desalination plant
}

\author{
V. Murugan, K. Rajanbabu, S.A. Tiwari, \\ C. Balasubramanian, Manoj Kumar Yadav, \\ A.Y. Dangore, S. Prabhakar* and P.K. Tewari \\ Bhabha Atomic Research Centre Facilities \\ Kalpakkam, 603102 India \\ E-mail: Tiwari-susati@magnum.barc.ernet.in \\ E-mail: Balasubramanian-cbs@igcar.ernet.in \\ E-mail: aydangore@igcar.ernet.in \\ E-mail: sprabha@apsara.barc.ernet.in \\ E-mail: Tewari-pktewari@ magnum.barc.ernet.in \\ *Corresponding author
}

\begin{abstract}
A seawater reverse osmosis plant of $1800 \mathrm{~m}^{3} /$ day capacity is part of the $6300 \mathrm{~m}^{3} /$ day capacity nuclear desalination demonstration project at Kalpakkam. The plant was commissioned in October 2002 and is in continuous operation. This paper deals with types of foulants, membrane cleaning procedures and the improvement in the reverse osmosis system after cleaning. This paper also describes the analysis of foulants, which may consist of adsorbed organic compounds, particulate matter, micro-organisms and metallic oxides, and describes the chemical cleaning procedure to be adopted, which is specific the seawater used because the membrane foulants are very specific with respect to the seawater constituents. The cleaning of the membranes in the Kalpakkam nuclear desalination plant was taken up because the quality of the permeate had deteriorated and the differential pressure across the membrane had gone up. This paper essentially deals with the selection of cleaning chemicals, the experience gained with the cleaning procedure adopted and the effects of cleaning on the membrane system.
\end{abstract}

Keywords: membrane cleaning; membrane fouling; reverse osmosis.

Reference to this paper should be made as follows: Murugan, V., Rajanbabu, K., Tiwari, S.A., Balasubramanian, C., Yadav, M.K., Dangore, A.Y., Prabhakar, S. and Tewari, P.K. (2006) 'Fouling and cleaning of seawater reverse osmosis membranes in Kalpakkam nuclear desalination plant', Int. J. Nuclear Desalination, Vol. 2, No. 2, pp.172-178.

Biographical notes: V. Murugan is Chemical Engineer and Scientific Officer at NDDP. He joined BARC in 1987 and has been associated with the activities of NDDP since 2000, having been involved in the building, commissioning and operation of the $1800 \mathrm{~m}^{3} / \mathrm{d}$ capacity seawater reverse osmosis plant at Kalpakkam.

K. Rajanbabu is Chemical Engineer and Scientific Officer at NDDP, Kalpakkam.

S.A. Tiwari is Chemical Engineer and Scientific Officer in the Desalination Division of Bhabha Atomic Research Centre (BARC). He is involved in the membrane-based desalination projects of the division. 


\section{Balasubramanian is Chemical Engineer and Scientific Officer at NDDP.}

Manoj Kumar Yadav is Mechanical Engineer and Scientific Officer at NDDP.

A.Y. Dangore graduated in Electrical Engineering from REC, Nagpur, and joined BARC training school in 1971. He has led a team of electrical and electronic engineers and has been responsible for the design of electrical systems for many important activities. For the last seven years, he has been Project Manager of NDDP, involved in the detailed engineering, construction, operation and maintenance of desalination plants. He is member of the Technical Advisory Committee constituted by Chennai Metro Water Supply and Sewarage Board for the proposed 100/200 MLD desalination plant.

S. Prabhakar holds a PhD in Membrane Science with a Master's degree in both Chemistry and Chemical Engineering. He has been involved in the development of reverse osmosis technology from membrane development stage to applications, both for desalination and effluent treatment, including radioactive effluents. He has experience in the design, installation and operation of reverse osmosis plants. He has been a part of IAEA activities as member of Coordinated Research Programs (CRP) He has more than 75 publications and three patents to his credit.

P.K. Tewari completed a PhD in Chemical Engineering from IIT, Mumbai, and is presently Head of the Desalination Division, BARC. He provides expert services to the International Atomic Energy Agency (IAEA), Vienna, for seawater desalination through CRP. He has provided consultancy services to a number of organisations on desalination and water reuse. He has more than 70 research publications to his credit in journals, proceedings, books and encyclopaedias. $\mathrm{He}$ is a recipient of an award from the International Desalination Association (IDA) $\mathrm{He}$ is member of the International Nuclear Desalination Advisory Group (INDAG).

This paper was presented at the Symposium on Desalination and Water Reuse, Trombay, India, 10-11 February 2005.

\section{Introduction}

Membrane fouling has become the most serious problem encountered in seawater reverse osmosis plants. Fouling refers to the deposition of undesirable material on the membrane surface, which affects the operation through a decline in the product water flux and/or increased salt passage and increased pressure drop between the feed and concentrated side. Fouling in reverse osmosis membranes is caused by inadequate pre-treatment system, upset pre-treatment conditions, improper material selection (pumps and piping, etc.) failure of the chemical dosing system, inadequate flushing following shutdown, improper operation control, slow buildup of precipitates over extended periods (barium, silica), change in feed water composition and biological contamination of feed water. Foulants are classified into four categories: adsorbed organic compounds, biological growth, metallic oxides and particulate matter. Generally, metallic oxides play a critical role in the cohesion of scales formed (or fouling layer). A primary foulant can inhibit the back diffusion of soluble ions and lead to secondary fouling, which has even more consequences. Organic matter and/or micro-organisms have become the main foulant in the membrane system. In addition, divalent cations $\left(e . g ., \mathrm{Ca}^{2+}\right)$ were proved to have a 
dramatic effect on fouling. Moreover, in seawater, micro-organisms preferring the membrane material best were found most commonly adhering to the membrane surface. Hence periodic cleaning of the membrane to remove foulant accumulations is essential to maintaining the quality and quantity of product water and to maximise membrane life. However, nearly all cleaning procedures are generalised by the membrane manufacturers, and also, the cleaning chemicals used for cleaning are proprietary in nature. This paper describes the study on the assessment of water chemistry, analysis of the foulant, the selection of chemicals for the cleaning procedure adopted and improved performance of the reverse osmosis plant after cleaning.

\section{Description of SWRO plant}

The cleaning of the membrane was done in the seawater reverse osmosis plant of the Nuclear Desalination Demonstration Project (NDDP) of Bhabha Atomic Research Centre Facilities, Kalpakkam, in the south of India. This seawater reverse osmosis plant shares facilities with Madras atomic power station, located next to its campus. This plant, with a capacity of $75 \mathrm{~m}^{3} / \mathrm{hr}$ of desalinated water, was commissioned in October 2002 by the Prime Minister of India, and is continuously supplying product water. The pre-treatment system of the reverse osmosis plant consists of the addition of a disinfectant through hypochlorite at the seawater intake pit. The flowsheet for this plant is given in Figure 1. The pre-treatment system of the reverse osmosis plant also involves Lamella clarifier, which clarifies the seawater to remove the total suspended solids from 400 to $40 \mathrm{ppm}$. The system involves the addition of ferrous sulphate as coagulant and polyelectrolyte as coagulant aid. Further, if required, shock dosing of disinfectant is done in the clarifier so that the residual chlorine at the outlet of the Pressure Sand Filter would be $0.3 \mathrm{ppm}$. Then clarified water is filtered in the pressure sand filter to reduce the total suspended solids concentration to a level of $<1 \mathrm{ppm}$. The water at the pressure sand filter outlet is again filtered in activated carbon filter to remove excess chlorine and organics, if any. Then the treated water is fed to high-pressure pumps through a five-cartridge filter. Besides filtration, chemicals like hydrochloric acid for controlling carbonate scaling, sodium bisulphite for ensuring nil chlorine at the exit of activated carbon filter, and sodium hexametaphosphate as antiscalant are added. The reverse osmosis unit consisting of SW8040HR membranes is being operated at 35\% recovery. A typical water analysis of raw seawater is listed in Table 1. The two-year record of foulant concentrations is shown in Figure 2.

Table 1 Typical analysis of raw seawater in NDDP, Kalpakkam

\begin{tabular}{lc}
\hline Components & Concentration ppm \\
\hline Calcium & 400 \\
Sodium & 10556 \\
Magnesium & 1272 \\
Potassium & 380 \\
Bicarbonates & 137 \\
Chlorides & 18981 \\
Sulphates & 2650 \\
Iron & 1.5 \\
\hline
\end{tabular}


Figure 11800 cu.m/day seawater reverse osmosis plant

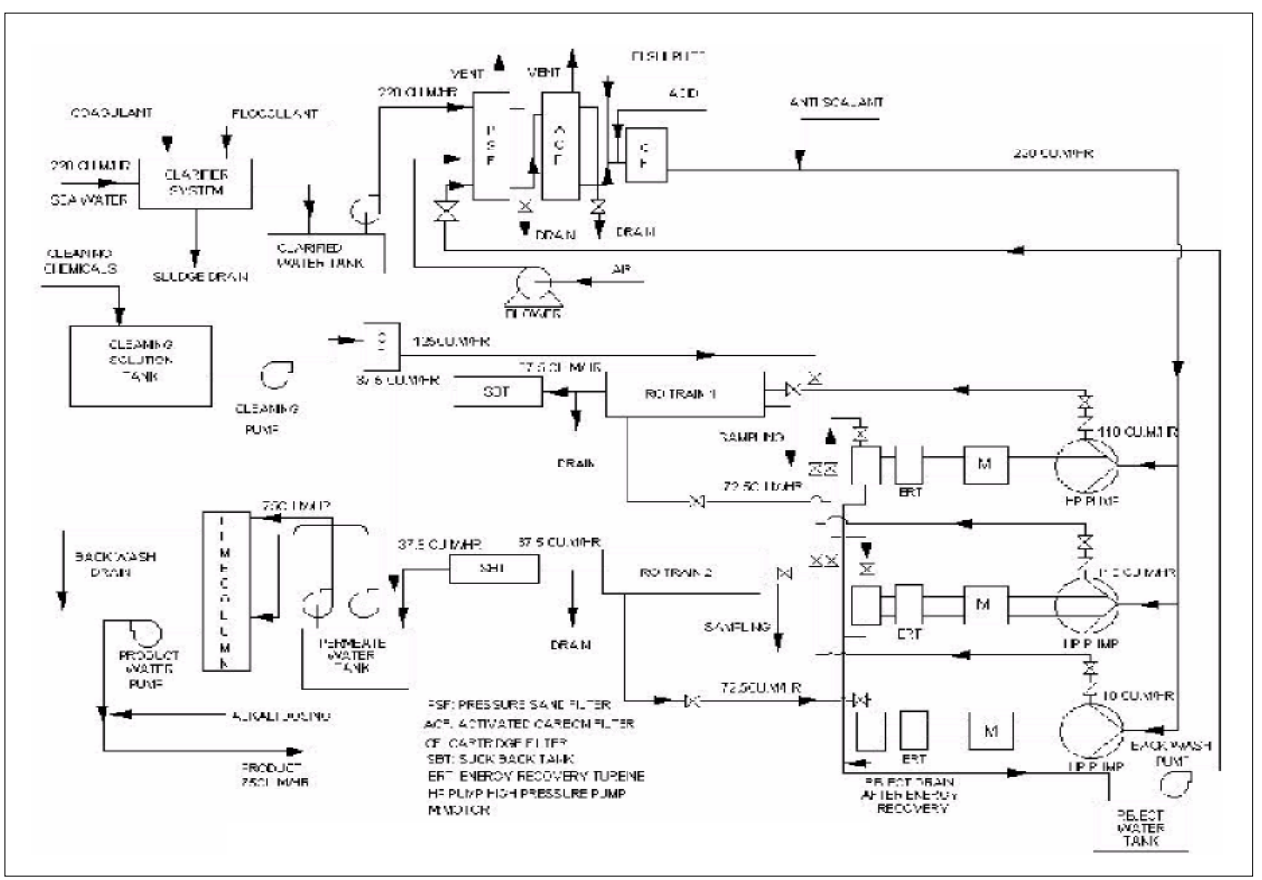

Figure 2 Concentration of foulants at various periods

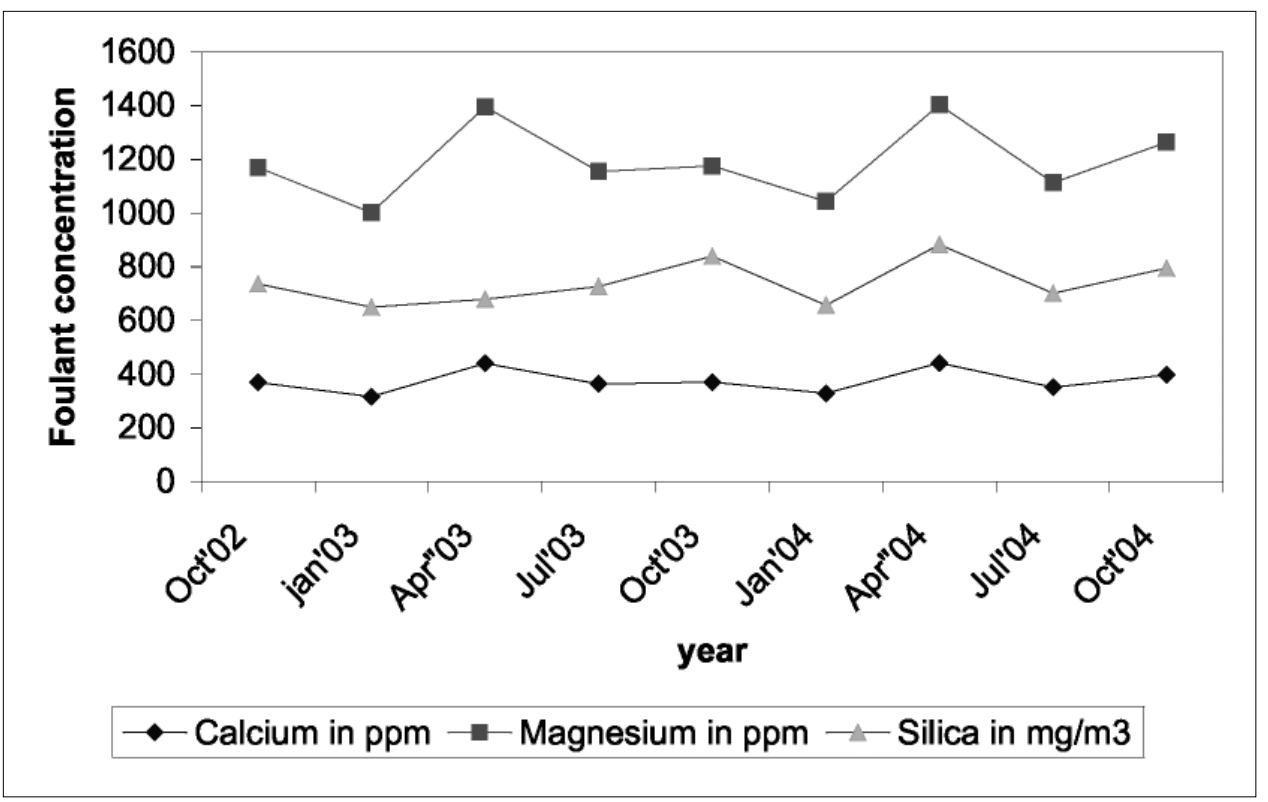


The raw seawater salinity and the turbidity vary with the season. During the monsoon, with active depression over the Bay of Bengal, the turbidity of seawater is comparatively higher. This can be seen from the statistical records of foulant concentrations given in Figure 2. Though the stringent pre-treatment system is in operation, avoiding the onset of fouling on the membrane surface is not possible. Owing to fouling, the plant performance has come down, which is shown in Table 2.

Table 2 Operational data of RO system

\begin{tabular}{lccc}
\hline Operational parameter & June 2003 & September 2004 & After cleaning \\
\hline Feed seawater & 33232 & 33105 & 32954 \\
Total dissolved solids in ppm & & & \\
Feed pressure $\mathrm{kg} / \mathrm{cm}^{2}$ & 40.6 & 44.0 & 41.0 \\
Feed flow $\mathrm{m}^{3} / \mathrm{h}$ & 110.5 & 110.2 & 110 \\
Recovery percentage (\%) & 35.1 & 35.0 & 35.1 \\
Rejection of salts percentage (\%) & 98.6 & 98.1 & 98.4 \\
Pressure drop across membranes & 0.789 & 0.950 & 0.829 \\
Permeate flow $\mathrm{m}^{3} / \mathrm{h}$ & 38.8 & 38.6 & 38.7 \\
\hline
\end{tabular}

\section{Selection of cleaning chemicals}

It was decided to take up the reverse osmosis system for cleaning, because the salt passage and pressure drop between the feed and concentrate side increased after sufficient operation, which are indications of the onset of fouling. The cleaning will be more effective if it is tailored to the specific fouling problem. Sometimes, a wrong choice of cleaning chemicals can even worsen the situation. Therefore, the types of foulant on the membrane surface should be determined prior to cleaning. From the analysis of precipitation on the 0.45 Millipore filter paper, it is understood that besides carbonate scaling, biofouling also would have occurred. So it was decided to first use $\mathrm{NaOH}$ cleaning, followed by acid cleaning.

Hydrochloric acid was selected over citric acid, as citric acid may act as a nutrient source for a system which has biological fouling, and is not as effective with thin-film composite chemistry.

\section{Cleaning procedure}

The concentration of the alkali solution was $0.1 \%(\mathrm{w} / \mathrm{v})$ and the $\mathrm{pH}$ of the solution was 11. The concentration of the acid solution was $0.5 \%$ and the $\mathrm{pH}$ of the solution was 2.5 .

\subsection{Alkali cleaning}

First, membranes were flushed with permeate water for about one hour and it was insured that no permeate water was held up in the permeate header. The alkali solution was prepared in the cleaning solution tank. The initial flow through the membrane modules 
was maintained at $40 \mathrm{~m}^{3} / \mathrm{h}$ and the highly fouled solution drained for two minutes. Then the solution was put in circulation for ten minutes. Whenever the $\mathrm{pH}$ decreased, it was raised by adding alkali and the flow was increased to $80 \mathrm{~m}^{3} / \mathrm{h}$ and in circulation for five minutes.

When the solution became hazy and turbid, the solution was discarded, fresh solution was prepared and slowly the flow was increased to $120 \mathrm{~m}^{3} / \mathrm{h}$ and kept in circulation for half an hour. Then the membranes were kept soaked for one hour. After soaking, the membranes were flushed with the permeate water until their conductivity and $\mathrm{pH}$ were the same as the permeate water.

\subsection{Acid cleaning}

The cleaning tank was well washed with the permeate water, the acid solution was prepared and cleaning was done similarly to the alkali wash except that the high-flow circulation was kept up for 1 hour. The cleaning solution samples were taken at different stages both during the acid and alkali wash. The results of the analysis of the cleaning solution for different foulant concentrations are given in Figure 3.

Figure 3 Concentration of foulants at various stages of cleaning

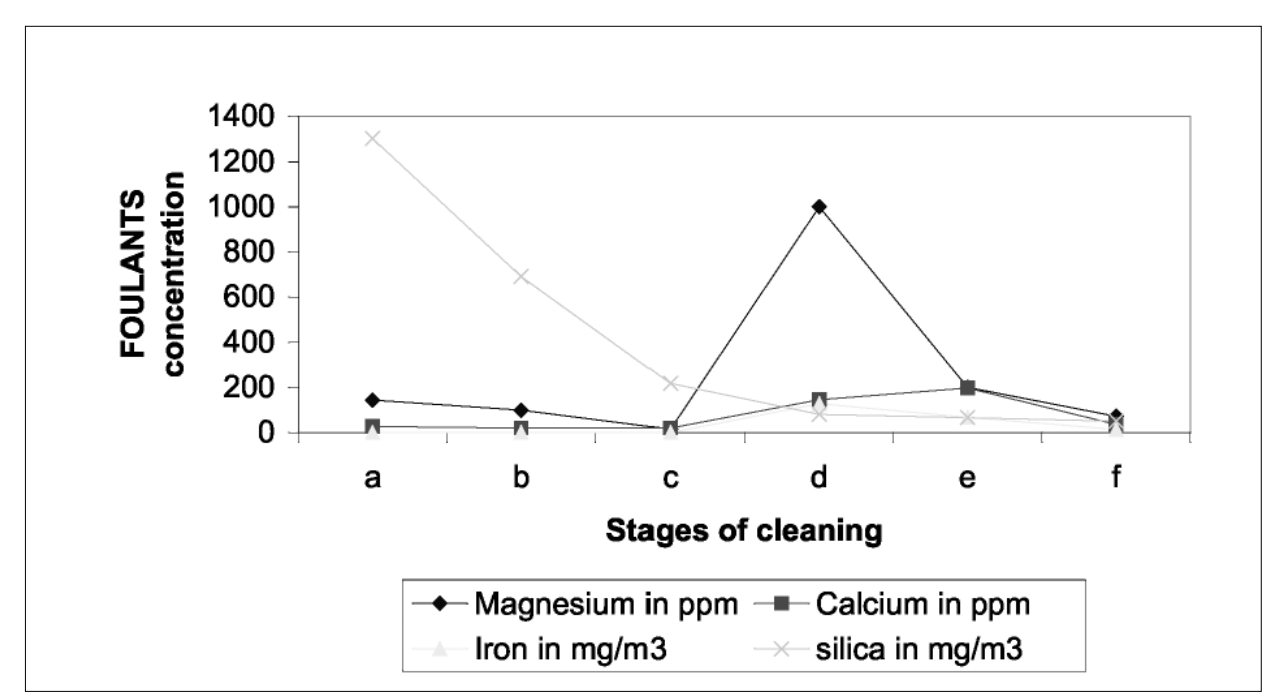

Notes: 1. a, b, c- represents alkali cleaning stages after ten minutes of alkali injection, one hour circulation and flushing respectively

2. $\mathrm{d}, \mathrm{e}, \mathrm{f}-$ represents acid cleaning stages after ten minutes of acid injection, one hour circulation and flushing respectively

\section{Results and discussions}

Table 2 gives performance data of the membranes before and after cleaning. It had been found that the rejection of salts had been reduced over a period of operation, which improved after the cleaning of the RO membrane for the given recovery period. Similarly, the pressure drop across the membrane module had increased over a period of 
operation, and was then reduced after the cleaning of the RO membranes. This shows that the chemical selection and the method of cleaning had been effective in improving the performance of the membranes.

\section{Acknowledgements}

The authors are grateful to Mr. D.S. Shukla, Director, Chemical Engineering and Technology Group, BARC, and S. Banerjee, Director, BARC for their support and encouragement, and to all those associated with the plant for their contributions. 